\title{
Using Coriolis Mass Flowmeter for Wet Gas Metering
}

\author{
Yanfeng Geng ${ }^{1}$, Hoi Yeung ${ }^{2}$, Yi Cao ${ }^{2}$, Lanchang Xing ${ }^{2}$, Hao Zhu ${ }^{3}$, Wolfgang Drahm ${ }^{3}$ \\ ${ }^{1}$ China University of Petroleum, ${ }^{2}$ Cranfield University, ${ }^{3}$ Endress+Hauser GmbH+Co.KG
}

Abstract

Established wet gas metering techniques are typically based on differential pressure devices, and their measurement accuracy is still unsatisfactory to natural gas industry. Coriolis mass flowmeter (CMF) is the most superior flow measurement technology at now, it can provide mass flowrate and density output simultaneously. Putting CMF into wet gas metering may be a reasonable and high accuracy solution to natural gas industry demands.

The problems and advantages of CMF in gas-liquid two-phase flow metering has been analyzed, a serious experiments has been carried out to test the possibility and feasibility of CMF as wet gas meter. Experimental conclusions are promising, CMF can work properly in wet gas metering condition, and the repeatability test of CMF is perfect, and the relationship between the true mass flowrate and CMF readings are almost linear. When gas flowrate is kept to a constant, CMF can follow the variations of liquid flowrate accurately. At last, some open problems deserving further investigation are proposed.

\section{Introduction}

Wet gas is the terminology for a well stream where the gas volume fraction (GVF) is greater than $90 \%$ and mostly above $95 \%$ at the metering condition. It is a typical gas-liquid two-phase flow with low liquid fractions. Currently, wet gas meter (WGM) is mainly based on differential pressure (DP) devices. At 90\% confidence level, the measurement uncertainties of existing WGMs are about 5 10\% for gas and liquid flowrate, which can meet the accuracy requirement for reservoir management, but cannot be used for production allocation and fiscal metering. The latter two accuracy requirements are $2 \sim 5 \%$ and $0.25 \sim 1 \%$ respectively. The development of high accuracy WGM is still a key demand to the natural gas industry. 
Due to the measurement uncertainty of DP meters is affected by fluid temperature, density, fluid velocity profile, fluid viscosity and so on; the accuracy of existing WGMs is very difficult to improve. Coriolis technology offers unprecedented accuracy and reliability in measuring single-phase flow. The accuracy of CMF is not affected by the above-mentioned facts. Letting CMF as WGM may be a reasonable solution to industry requirement ${ }^{[1]}$.

\section{Problems of CMF in gas-liquid two-phase flow metering}

Early experimental results from Micro Motion showed that CMF was unable to perform well when presented with gas-liquid two-phase flow. The main effect is a dramatic rise in the flowtube damping, and not only does the damping rise, but it vary rapidly, due to the chaotic nature of the interactions among liquid, compressible gas and flowtube walls. This phenomenon directly leads the flowtube drive circuit to saturation, as the drive current is used for overcoming the damping effect, and maintaining the flowtube to vibrate in constant amplitude. Rapid changes in damping will result in rapid changes in the parameters of flowtube vibration, which include frequency, amplitude and phase signals from CMF sensors. And then the changes of sensor signals increase the difficulties of useful information extraction, and finally lead to poor tracking of frequency and phase, and proper drive current cannot be generated in time. There will be phase shift between sensor input and drive output existing at this moment, the flowtube may stall or vibrate in a forced mode. The accuracy and repeatability of CMF are significantly degraded in gas-liquid two-phase flow metering.

Recently, there are many improvements in CMF technology ${ }^{[2]}$, which includes CMF tube geometry design based on numerical simulations, tube balancing system, and signal processing techniques and so on. It's time to reconsider the possibility and feasibility for multiphase flow metering based on CMF technology. The optimal CMF operation is the flowtube should vibrate at its natural frequency with constant amplitude. The vibrating frequency of flowtube is a function of fluid density, and the phase 
difference from the two CMF sensors is a function of mass flowrate. In practice, there are two problems needed to be solved before putting CMF into wet gas metering. The first one is to maintain the flowtube vibrating as stable as possible. The second one is to get accurate mass flow and density readings from sensor signals.

\section{Theoretical analysis for CMF as WGM}

In case of wet gas flow metering, the Coriolis tube will have to endurance complicated force from two-phase fluid in different flow regimes. So, the simpler the tube is, the less additional forces the tube will endurance. Supposing the diameter of Coriolis tube is in the same size with the connected pipeline, the tube will have no influence on flow regime, and hence no additional force arose by two-phase fluid on Coriolis tube. So, the straight tube Coriolis meter would be the best choice for wet gas flow metering. The force from two-phase fluid is added up with Coriolis force, it is almost impossible to distinguish or separate them.

The added forces are the main sources of high degree noise in CMF sensor signals.

On the other hand, there are some disadvantages for straight tube CMF as single-phase flowmeter. Compared with a typical bent flowtube, the sensor signals arising from a straight tube have a high-frequency, low-amplitude, and low-phase difference range. In other words, they have a poorer signal-to-noise ratio. And it is more difficult than bent tubes to extract good quality measurement signals and ensure continuous flowtube oscillation. But in our experiments of CMF as WGM, the signal to noise ratio is not as high as bent tube CMF reported in Ref.3. In our study, two slightly bent tube CMFs were selected and tested, they show good performances as illustrated below, and they can work properly through all the testing range.

Furthermore, one of the main problems of existing WGMs is that their turndown ratio is less than 10 to 1 . But the total mass flowrate of wet gas in the production pipeline always varies more than one hundred times due the natural properties of gas-liquid two-phase flow, so the DP reading is saturated randomly. 
Straight tube CMF has a turndown ratio of 1000 to 1 , it can deal with the natural variation of gas-liquid flowrate easily. In this view, straight tube CMF is the optimal selection for wet gas metering.

In theory, concerning with the two problems in practice, there will be three steps needed to make CMF into WGM. The first step is modeling CMF in wet gas flow metering; the traditional model for CMF is not suitable because the compressibility of gas phase will lead to different vibrations among gas, liquid and flowtube walls. Especially in different flow regimes, such as stratified flow, annular flow and mist flow, the CMF metering model must be different. The second step is the developing of model-based control method in order to keep the flowtube's vibration parameters following gas-liquid two-phase flowrate variation in a stable, steady and fast way. The last step is the developing of model-based signal processing algorithm in order to obtain accurate gas and liquid flowrate.

\section{Laboratory test and some results}

Experiments has been carried out in the two-phase flow experimental rig in Cranfield University, UK. The detail description of the rig can be found in Ref.4. The test matrix is showed in fig. 1. Two selected CMFs were installed in sequence in the rig, one is in vertical installation, and the other is in horizontal. The diameters of experimental pipeline and $\mathrm{CMF}$ are 2 inches. The experimental fluid are air-water and air-oil. The gas flowrate is $200 \sim 500 \mathrm{sm}^{3} \mathrm{~h}^{-1}$, liquid flowrate is $0.11 \sim 1.5 \mathrm{Kgs}^{-1}$, and pressure is $100 \sim 300 \mathrm{KPa}$. The calculated GVF in metering condition is $88 \%$ 99\%, which are in the range of wet gas flow. In order to be

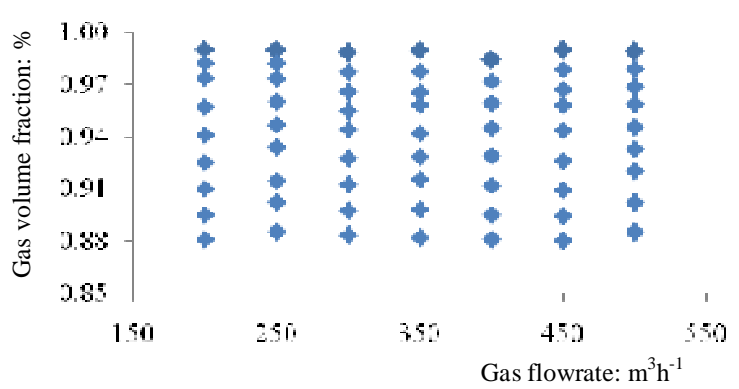

Fig 1 Wet gas test matrix concise in the following paragraph, we call the vertical coriolis meter CMF01, and the horizontal one CMF02. Except for comparision of the effects of two kinds of installation, the results will be mainly focus on CMF02. The sampled data include amplitude and frequency of flowtube vibration, total mass flowrate, exicitor current, damping coefficient, temperature of fluid, temperature of flowtube and DP signals across CMF. Based on the experimental data analysis, the following results can be obtained.

The first result of CMF as WGM is that the measurement repeatability is quite good although the magnitude of the errors is high. Table 1 shows the repeatability test results of frequency reading. The 
repeatability error is defined as the relative error of frequency output; the frequency output range is $465 \mathrm{~Hz} \sim 562 \mathrm{~Hz}$, the upper and low limits are CMF frequency for gas and liquid single phase flow measurement respectively. The repeatability test was carried out at different time but with almost the same gas and liquid flowrate, same pressure and temperature. The two times frequency output are indexed by Frequency No.1 and Frequency No.2.

The selected wet gas flowrate in table 1 has almost the same GVF in the metering condition, and hence the flowtube has almost the same oscillating frequency. The biggest repeatability error is less than $0.5 \%$, and most of them are less than $0.2 \%$. It is quite good for WGM in industry, and this is a potential advantage of CMF as WGM over other kind of flow meters and density meters.

Table 1 Repeatability test results of CMF02 frequency reading for wet gas flow

\begin{tabular}{|l|c|c|c|c|c|c|c|}
\hline Gas flow & $200 \mathrm{sm}^{3} \mathrm{~h}^{-1}$ & $250 \mathrm{sm}^{3} \mathrm{~h}^{-1}$ & $300 \mathrm{sm}^{3} \mathrm{~h}^{-1}$ & $350 \mathrm{sm}^{3} \mathrm{~h}^{-1}$ & $400 \mathrm{sm}^{3} \mathrm{~h}^{-1}$ & $450 \mathrm{sm}^{3} \mathrm{~h}^{-1}$ & $500 \mathrm{sm}^{3} \mathrm{~h}^{-1}$ \\
\hline Liquid flow & $0.70 \mathrm{Kgs}^{-1}$ & $0.98 \mathrm{Kgs}^{-1}$ & $0.99 \mathrm{Kgs}^{-1}$ & $1.20 \mathrm{Kgs}^{-1}$ & $1.70 \mathrm{Kgs}^{-1}$ & $2.00 \mathrm{Kgs}^{-1}$ & $2.40 \mathrm{Kgs}^{-1}$ \\
\hline Frequency No.1 & $556.68 \pm 1.21$ & $557.04 \pm 0.95$ & $557.63 \pm 0.77$ & $557.53 \pm 0.66$ & $557.07 \pm 0.72$ & $556.99 \pm 0.67$ & $556.70 \pm 0.62$ \\
\hline Frequency No.2 & $556.29 \pm 1.13$ & $556.88 \pm 0.93$ & $557.36 \pm 0.76$ & $557.54 \pm 0.64$ & $557.19 \pm 0.67$ & $557.02 \pm 0.61$ & $556.76 \pm 0.60$ \\
\hline Repeatability error & $0.40 \%$ & $0.16 \%$ & $0.28 \%$ & $0.01 \%$ & $0.12 \%$ & $0.03 \%$ & $0.06 \%$ \\
\hline
\end{tabular}

The sencond one is that the internal parameter of CMF varies rapidly during measurement process. Table 2

shows the mean value and standard deviation of the frequency, excitor current and damping coefficient in three different metering conditions, they are gas only, liquid only and wet gas metering. In table 2, gas flowrate is $150 \mathrm{sm}^{3} \mathrm{~h}^{-1} \sim 700 \mathrm{sm}^{3} \mathrm{~h}^{-1}$, pressure is $300 \mathrm{KPa}$, the temperature is $12^{\circ} \mathrm{C}$, liquid flowrate is $0.2 \mathrm{kgs}^{-1} \sim 7.1 \mathrm{kgs}^{-1}$. Wet gas flow is consisted of $350 \mathrm{sm}^{3} \mathrm{~h}^{-1}$ air flow and $0.45 \mathrm{kgs}^{-1}$ water flow, the calculated GVF is $97.7 \%$.

Table 2 Internal parameter of CMF for single-phase flow and wet gas flow

\begin{tabular}{|l|l|l|l|}
\hline \multicolumn{1}{|c|}{ Parameters } & \multicolumn{1}{c|}{ Gas flowrate } & \multicolumn{1}{c|}{ Liquid flowrate } & \multicolumn{1}{c|}{ Wet gas flow } \\
\hline Frequency of CMF01 & $562.185 \pm 0.112$ & $465.230 \pm 0.005$ & $558.818 \pm 0.477$ \\
\hline Frequency of CMF02 & $562.648 \pm 0.108$ & $465.097 \pm 0.004$ & $559.178 \pm 0.441$ \\
\hline Excitor crrent of CMF01 & $3.047 \pm 0.099$ & $3.358 \pm 0.047$ & $63.275 \pm 6.893$ \\
\hline Excitor crrent of CMF02 & $3.179 \pm 0.200$ & $3.389 \pm 0.023$ & $64.863 \pm 7.177$ \\
\hline Damping coefficient of CMF01 & $316.01 \sim 445.06$ & $345.46 \sim 426.15$ & $6805.74 \pm 800.40$ \\
\hline Damping coefficient of CMF02 & $321.05 \sim 517.51$ & $345.30 \sim 394.47$ & $6798.94 \pm 781.75$ \\
\hline
\end{tabular}

Coriolis meter can work properly in the testing process, but it can be seen from table 2 that the internal parameters varies violently in wet gas metering condition, the standard deviation of frequency varies 4 times to that of gas flow, and it is about 100 times to that of liquid flow. These phenomenon arise new and high requiremnet to signal processing and control method for CMF as WGM. 
The third one is that there are great differences between the two kinds of installation for wet gas metering while there is little differences for single-phase flow measurement. Generally speaking, The vertical installation is preferable for multiphase flowmeters which are based on gama ray or electrical sensors to obtain the fraction measurement. As in vertical pipeline the flow regime is simple and flow distribution across the pipe is uniform, vertical installation is helpful to get accurate fraction measurement. The horizontal installation is preferable for multiphase flowmeters based on DP technology, as in the vertical pipeline the DP readings arosed from gravity and throttlling are difficult to separate each other, but the gravity has no influences on DP measurement in horizontal installation.

Fig 2 shows two groups of mass flowrate signals from CMF01 and CMF02 for liquid flow and wet gas flow measurement. Liquid flowrate is $0.45 \mathrm{Kgs}^{-1} \sim 0.70 \mathrm{Kgs}^{-1}$. Wet gas flow is consisted of $200 \mathrm{sm}^{3} \mathrm{~h}^{-1}$ gas flowrate and $0.116 \mathrm{Kgs}^{-1}$ water flowrate. The conclusions from fig. 2 are: (1). In the single-phase flow measurement, there is almost no differences between the two CMF outputs in fig.2(a). As CMF01 is installed in front of CMF02, there is a time

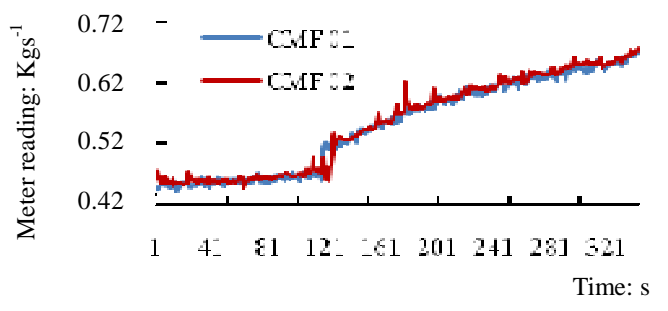

(a) Liquid flow metering of CMF01 and CMF02

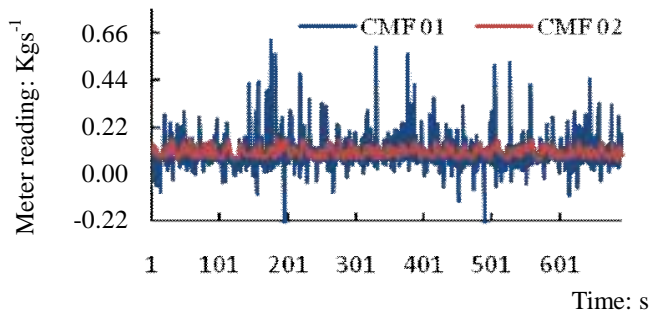

(b) Wet gas metering of CMF01 and CMF 02

Fig 2 Comparison of CMF01 and CMF02 difference between the two meter's output signals. (2.there are violent variations in the output signals from CMF01, and some of its mass flow readings are negtive, so the horizontal installation is preferable for wet gas metering. Actually after the gas flowrate has been increased over $250 \mathrm{sm}^{3} \mathrm{~h}^{-1}$ (liquid flowrate is kept to $\left.0.226 \mathrm{Kgs}^{-1}\right)$, all the reading from CMF01 is positive, but the output variation of CMF01 is still bigger than that of CMF02. (3). Theoretical analysis and experimental data all showes that when gas flowrate is less than 250 $\mathrm{sm}^{3} \mathrm{~h}^{-1}$ and liquid flowrate is greater than $0.11 \mathrm{Kgs}^{-1}$, there will be an inverse liquid flow exist in the vertical pipeline, also in CMF01, it is the main reason for negtive output of CMF01. 
The fourth one is about density measurement of wet gas flow. Fig 3 shows the density reading from CMF02.

The true density was calculated by gas and liquid flowrate from references meter in the experimental rig. Some conclusion can be made from fig 3: (1). If gas flowrate is kept to a constant, the relationship between true density and density reading is approximately linear. That is to say coriolis

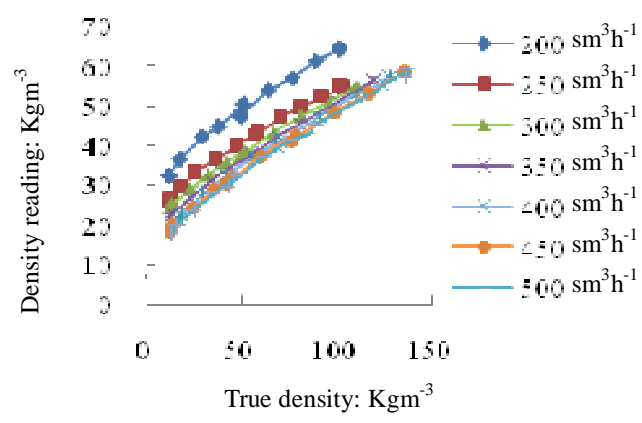

Fig 3 Density reading and real density value meter can following the variation of liquid fractions accurately when gas flowrete is known. (2). The slope and intercept is varying with gas and liquid flowrate. The reason behind this variation deserves further investigation.

(3). After gas flowrate is greater than $350 \mathrm{sm}^{3} \mathrm{~h}^{-1}$, the slope and intercept will become close to each other.

The fifth one is about the total mass flowrate measurement of wet gas flow. In the all air/water experimental ranges, CMF01 mass flow reading and true mass flow is showed in fig 4 . It can be seen that there is

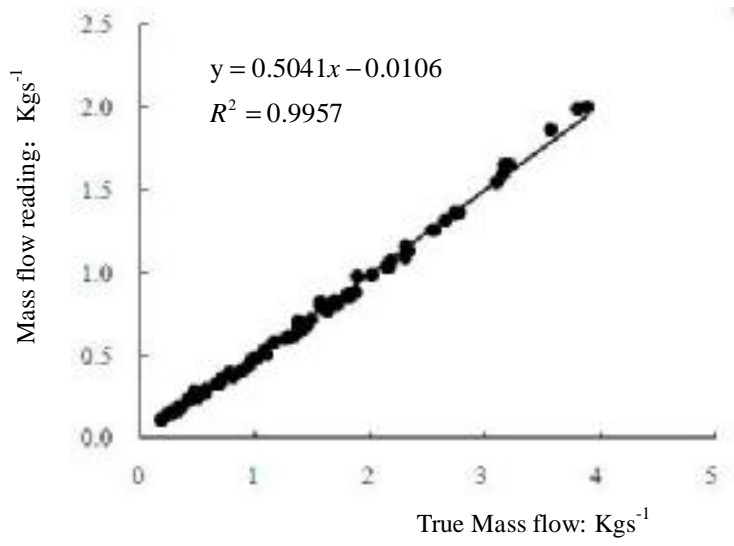

Fig 4 Mass flow reading and true mass flowrate an almost linear relationship between them, the coefficient of determination is 0.9957 . It is a good news for the correction of CMF readings, and it provides the possibility for high accuracy measurement of wet gas flow. The facts which have influences on slope and interception of linear relationship are needed to be investigated in the future.

5. Conclusion and future works

(1). There are many advantages of CMF as WGM, the most important two facts are the relationship between the true mass flowrate and CMF readings is almost linear, and CMF can following the variation of liquid flowrate linearly when gas flowrate is kept to a constant.

(2). For CMF as WGM in a wide range of measurement, the horizontal installation will be more preferable. 
When gas flowrate is big enough and liquid flowrate is low, the differences between the two installations will disappear.

(3). Perfact repeatability, properly working and linear relationship makes it possible and feasible for CMF as WGM. Medel first and model-based control second, model-based signal processing thirdly is a reasonable solution to this problem.

Acknowledgement: Thanks to national natural science foundation of China support (60672003), and thanks the engineers from Endress+Hauser for their technique support in the modification of meter's parameter and control algorithm for this special research.

\section{References}

(1). Robbie L., Jacob S., Michael R., etc. Coriolis mass flow metering for wet gas, Measurement + Control, 41:213-216, 2008

(2). Martin A., Wolfgang D. etc. Coriolis mass flow meters: overview of current state of the art and latest research, Flow Meas. Instr., 17(6), 317-323, 2006

(3). Henry M. P., Tombs M., Duta M.D., etc. Two-phase flow metering of viscous oil using a coriolis meter: a case study, Flow measurement and Instrumentation, 17:399-413, 2006

(4). Yi Cao, Hoi Yeung, Introduction to the experimental rig in Cranfield university, 2008 